\title{
Homogenization Closure For A Two-Dimensional Effective Model Describing Fluid-Structure Interaction in Blood Flow
}

\author{
Andro Mikelić ${ }^{1}$ and Sunčica Čanić ${ }^{2}$ \\ 1 Institut Camille Jordan, UFR Mathématiques \\ Université Claude Bernard Lyon 1, Site de Gerland, Bât. A, \\ 50, avenue Tony Garnier, 69367 Lyon Cedex 07, France \\ Andro.Mikelic@univ-lyon $1 . \mathrm{fr}^{\dagger}$ \\ 2 Department of Mathematics \\ University of Houston, 4800 Calhoun Rd. \\ Houston TX 77204-3476, United States \\ canic@math.uh.edu
}

\section{Introduction}

We study the flow of a viscous incompressible fluid through a long and narrow elastic tube whose walls are modeled by the Navier equations for a curved, linearly elastic membrane.

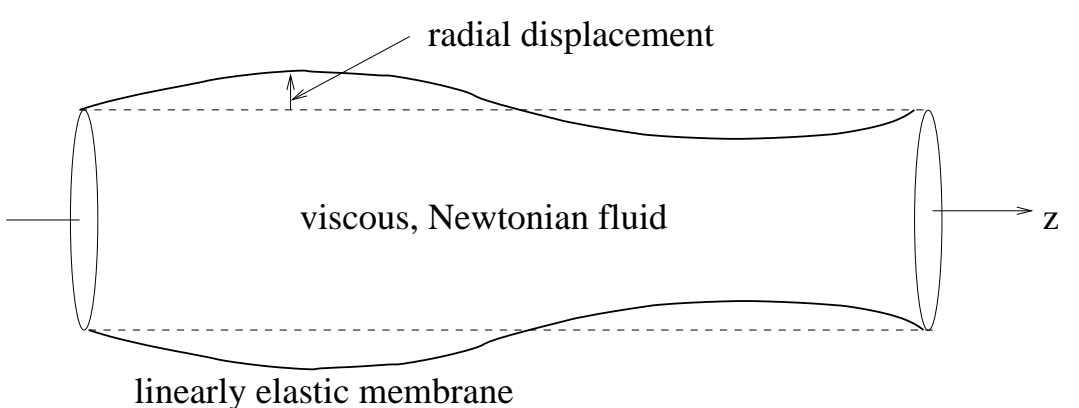

Fig. 1. Domain Sketch

The flow takes place in $\Omega_{\varepsilon}=\left\{x \in \mathbb{R}^{3} ; x=(r \cos \vartheta, r \sin \vartheta, z), r<\right.$ $\left.R+\eta^{\varepsilon}(z, t), 0<z<L\right\}$ and is governed by a given time dependent pressure drop between the inlet and the outlet boundary, giving rise to a non-stationary in-

\footnotetext{
${ }^{\dagger}$ This paper is dedicated to Professor Vincenzo Capasso for his 60th birthday
} 
compressible flow modeled by the Navier-Stokes equations. The aspect ratio $\varepsilon=\frac{R}{L}$ is " small " $\left(\approx 3 \cdot 10^{-2}\right)$.

We suppose that the lateral boundary of the cylinder $\Sigma_{\varepsilon}=\left\{r=R+\eta^{\varepsilon}\right\} \times$ $(0, L)$ behaves as a linearly elastic membrane of thickness $h$, that the longitudinal displacement is zero, and that the radial displacement satisfies Navier's equation

$$
-F_{r}=\frac{h(\varepsilon) E(\varepsilon)}{1-\sigma^{2}} \frac{\eta^{\varepsilon}}{\varepsilon^{2} R^{2}}+p_{r e f} \frac{\eta^{\varepsilon}}{R}-h(\varepsilon) G(\varepsilon) k(\varepsilon) \frac{\partial^{2} \eta^{\varepsilon}}{\partial z^{2}}+\rho_{w} h(\varepsilon) \frac{\partial^{2} \eta^{\varepsilon}}{\partial t^{2}},
$$

In (1), $\eta^{\varepsilon}$ is the radial displacement from the reference state in Lagrangian coordinates (see Figure 1), $h=h(\varepsilon)$ is the membrane thickness, $\rho_{w}$ the wall volumetric mass , $E=E(\varepsilon)$ is the Young modulus, $0<\sigma<1$ is the Poisson ratio, $G=G(\varepsilon)$ is the shear modulus and $k=k(\varepsilon)$ is Timoshenko shear correction factor (see [QTV:00]). $F_{r}$ is the radial component of the external forces, coming from the stresses induced by the fluid, given by

$$
-F_{r}=\left(\left(p^{\varepsilon}-p_{r e f}\right) I-2 \mu D\left(v^{\varepsilon}\right)\right) \mathbf{n} \mathbf{e}_{r}\left(1+\frac{\eta^{\varepsilon}}{R}\right) \sqrt{1+\left(\frac{\partial \eta^{\varepsilon}}{\partial z}\right)^{2}} .
$$

where $D\left(v^{\varepsilon}\right)$ is the rate of strain tensor. Equation (2) is valid on $\Sigma_{\varepsilon}$. At the wall $\Sigma_{\varepsilon}$ we require continuity of velocity: the fluid velocity $v^{\varepsilon}$ is linked with the velocity of the lateral wall $\Sigma_{\varepsilon}$ by

$$
v_{r}^{\varepsilon}\left(R+\eta^{\varepsilon}, z, t\right)=\frac{\partial \eta^{\varepsilon}}{\partial t} ; \quad \text { and } \quad v_{z}^{\varepsilon}\left(R+\eta^{\varepsilon}, z, t\right)=0 \forall t \in \mathbb{R}_{+} .
$$

A time-dependent pressure head data at the inlet and at the outlet boundary drive the problem and we assume the following initial and boundary conditions

$$
\begin{array}{r}
p^{\varepsilon}+\rho\left(v_{z}^{\varepsilon}\right)^{2} / 2=P_{j}(t)+p_{r e f}, \text { with } j=1 \text { for } z=0, j=2 \text { for } z=L, \forall t \\
\qquad\left.v_{r}^{\varepsilon}\right|_{z=0, L}=0, \eta^{\varepsilon}=0 \text { for } z=0, \eta^{\varepsilon}=0 \text { for } z=L, \forall t .
\end{array}
$$

We will assume that the pressure $\operatorname{drop} A(t)=P_{1}(t)-P_{2}(t) \in C_{0}^{\infty}(0,+\infty)$.

The Eulerian formulation of an incompressible viscous flow is given by the axially symmetric Navier-Stokes equations for $\mathbf{v}^{\varepsilon}=\left(v_{r}^{\varepsilon}, v_{z}^{\varepsilon}\right)$ and $p^{\varepsilon}$ :

$$
\rho\left\{\frac{\partial \mathbf{v}^{\varepsilon}}{\partial t}+\left(\mathbf{v}^{\varepsilon} \nabla\right) \mathbf{v}^{\varepsilon}\right\}-\mu \Delta \mathbf{v}^{\varepsilon}+\nabla p^{\varepsilon}=0 \quad \text { in } \Omega_{\varepsilon} \times \mathbb{R}_{+},
$$

Initially, the cylinder is filled with fluid and the entire structure is in an equilibrium. The equilibrium state has an initial reference pressure $P_{0}=p_{\text {ref }}$ and the initial velocity zero. Furthermore, the initial data are given by

$$
\eta^{\varepsilon}=\frac{\partial \eta^{\varepsilon}}{\partial t}=0 \quad \text { on } \quad \Sigma_{\varepsilon}(0) \times\{0\} .
$$

We study the behavior of this coupled fluid-structure system (1)-(7) in the limit when $\varepsilon \rightarrow 0$. We derive the asymptotic equations that describe: (a) the flow occurring at 
the leading order time scale and (b) the oscillations of the membrane caused by a response of the elastic material. Since they occur at different time scales we introduce the scaling $\tilde{t}=\omega^{\varepsilon} t$. Classical 1D models lead to the variants of the shallow water model and require an ad hoc closure assumption. In this paper we will present effective equations which are obtained using homogenization, from the system (1)-(7), in the limit $\varepsilon \rightarrow 0$, without making any ad hoc assumptions.

\section{Uniform a priori estimates}

First we note that existence of solutions to the system (1)-(7) is an open problem. Recent references, containing existence results for the short time/small data can be found in [DEGLT:01] and [BdV:04].

We suppose existence of a smooth solution and study the energy estimate. The energy estimate, containing precise dependence on $\varepsilon$, is obtained in [CMT:05]. In order to capture the waves of the coupled fluid-structure response to the outside forcing, the authors introduced the new time $\tilde{t}=\omega^{\varepsilon} t$. The characteristic frequency $\omega^{\varepsilon}$ is calculated in [CLMT:05] by requiring that the effects of both the pressure head data, $P_{1}(t)$ and $P_{2}(t)$, as well as the pressure drop data, $A(t)$, are seen in the solution. It was found that

$$
\tilde{t}=\omega^{\varepsilon} t ; \quad \omega^{\varepsilon}=\frac{1}{L} \sqrt{\frac{R \mathcal{C}}{2 \rho}}
$$

Notice that $c=L \omega^{\varepsilon}$ is the characteristic wave speed (the local pulse wave velocity or sound speed). Expression (8) leads to the same characteristic wave speed as in [Fung:93].

We start by introducing the norms that will be used to measure the size of the inlet and the outlet boundary data. Define

$$
\begin{gathered}
\mathcal{C}=\frac{h(\varepsilon) E(\varepsilon)}{R^{2}\left(1-\sigma^{2}\right)}\left(1+\frac{p_{\text {ref }}}{R(\varepsilon)} \frac{R}{h(\varepsilon)}\left(1-\sigma^{2}\right)\right) ; \quad \hat{P}=\frac{A(t)}{L} z+P_{1}(t) \\
\left.\mathcal{P}^{2} \equiv \sup _{z, t}|\tilde{P}|^{2}+\sup _{z} \int_{0}^{t}\left|\frac{\partial}{\partial t} \hat{P}\right| d \tau\right)^{2}+T \int_{0}^{t}|A(\tau)|^{2}
\end{gathered}
$$

Using the precise energy inequality, in [CMT:05] the following a priori estimates were obtained:

Proposition 1. Solution $\left(v_{r}^{\varepsilon}, v_{z}^{\varepsilon}, \eta^{\varepsilon}\right)$ of problem (1)-(7) satisfies the following a priori estimates

$$
\begin{gathered}
\frac{1}{L}\left\|\eta^{\varepsilon}(\tilde{t})\right\|_{L^{2}(0, L)}^{2} \leq \frac{32}{\mathcal{C}^{2}} \mathcal{P}^{2} \\
\frac{1}{L R^{2} \pi}\left\|v^{\varepsilon}\right\|_{L^{2}\left(\Omega_{\varepsilon}(\tilde{t})\right)}^{2} \leq \frac{32}{\rho R \mathcal{C}} \mathcal{P}^{2} \\
\int_{0}^{\tilde{t}}\left\{\left\|\frac{\partial v_{r}^{\varepsilon}}{\partial r}\right\|_{L^{2}\left(\Omega_{\varepsilon}(\tilde{t})\right)}^{2}+\left\|\frac{\partial v_{z}^{\varepsilon}}{\partial z}\right\|_{L^{2}\left(\Omega_{\varepsilon}(\tilde{t})\right)}^{2}\right\} d \tau \leq \frac{4 \pi R^{2}}{\mu} \sqrt{\frac{2}{\rho R \mathcal{C}}} \mathcal{P}^{2}
\end{gathered}
$$




$$
\int_{0}^{\tilde{t}}\left\{\left\|\frac{\partial v_{z}^{\varepsilon}}{\partial r}\right\|_{L^{2}\left(\Omega_{\varepsilon}\right)}^{2}+\left\|\frac{v_{r}^{\varepsilon}}{r}\right\|_{L^{2}\left(\Omega_{\varepsilon}(\tilde{t})\right)}^{2}+\left\|\frac{\partial v_{r}^{\varepsilon}}{\partial z}\right\|_{L^{2}\left(\Omega_{\varepsilon}\right)}^{2}\right\} d \tau \leq \frac{4 R^{2}}{\mu} \sqrt{\frac{2}{\rho R \mathcal{C}}} \mathcal{P}^{2}
$$

The a priori estimates (11)-(14) provide a basis for asymptotic analysis in terms of the parameters of the problem.

Table 1. Table with parameter values

\begin{tabular}{lll}
\hline PARAMETERS & \multicolumn{2}{l}{ AORTA/ILIACS LATEX TUBE } \\
\hline Char. radius $R(\mathrm{~m})$ & $0.006-0.012$ & 0.011 \\
\hline Dyn. viscosity $\mu\left(\frac{\mathrm{kg}}{\mathrm{ms}}\right)$ & $3.5 \times 10^{-3}$ & $3.5 \times 10^{-3}$ \\
\hline Young's modulus $\mathrm{E}(\mathrm{Pa})$ & $10^{5}-10^{6}$ & $1.0587 \times 10^{6}$ \\
\hline Wall thickness $\mathrm{h}(\mathrm{m})$ & $1-2 \times 10^{-3}$ & 0.0009 \\
\hline Wall density $\rho_{S}\left(\mathrm{~kg} / \mathrm{m}^{2}\right)$ & 1.1, & 1.1 \\
\hline Fluid density $\rho\left(\mathrm{kg} / \mathrm{m}^{3}\right)$ & 1050 & 1000 \\
\hline
\end{tabular}

\section{From Asymptotic Expansions to Reduced Equations}

\subsection{Asymptotic expansion}

Introduce the non-dimensional independent variables $\tilde{r}$ and $\tilde{z}$

$$
r=R \tilde{r}, \quad z=L \tilde{z},
$$

and recall that the time scale for the problem is determined by $t=\frac{1}{\omega^{\varepsilon}} \tilde{t}$. Based on the a priori estimates, we introduce the following asymptotic expansions

$$
\begin{gathered}
v^{\varepsilon}=V\left\{\tilde{v}^{0}+\varepsilon \tilde{v}^{1}+\ldots\right\}, V=\sqrt{\frac{1}{R \rho \mathcal{C}}} \mathcal{P} \\
\eta=\Xi\left\{\tilde{\eta}^{0}+\varepsilon \tilde{\eta}^{1}+\ldots\right\}, \Xi=\frac{1}{\mathcal{C}} \mathcal{P} \\
p=\rho V^{2}\left\{\tilde{p}^{0}+\varepsilon \tilde{p}^{1}+\ldots\right\} .
\end{gathered}
$$

The approximate values of the scaling parameters, based on our parameters with $E=6 \times 10^{5} \mathrm{~Pa}$ are $V=0.5 \mathrm{~m} / \mathrm{s}, \omega=113$ and $\Xi=0.00025 \mathrm{~m}$.

After ignoring the terms of order $\varepsilon^{2}$ and smaller, the leading-order asymptotic equations describing the conservation of axial and radial momentum, and the incompressibility condition in non-dimensional variables read

$$
\begin{aligned}
S h \frac{\partial \tilde{v}_{z}}{\partial \tilde{t}}+\tilde{v}_{z} \frac{\partial \tilde{v}_{z}}{\partial \tilde{z}}+\tilde{v}_{r} \frac{\partial \tilde{v}_{z}}{\partial \tilde{r}}+\frac{\partial \tilde{p}}{\partial \tilde{z}}-\frac{1}{R e}\left\{\frac{1}{\tilde{r}} \frac{\partial}{\partial \tilde{r}}\left(\tilde{r} \frac{\partial \tilde{v}_{z}}{\partial \tilde{r}}\right)\right\}=0, \\
\frac{\partial \tilde{p}}{\partial \tilde{r}}=0, \quad \frac{\partial}{\partial \tilde{r}}\left(\tilde{r} \tilde{v}_{r}\right)+\frac{\partial}{\partial \tilde{z}}\left(\tilde{r} \tilde{v}_{z}\right)=0,
\end{aligned}
$$


where $\tilde{v}_{r}^{0}=0$ and

$$
S h:=\frac{L \omega^{\varepsilon}}{V}, R e:=\frac{\rho V R^{2}}{\mu L} ; \quad \tilde{v}_{r}=\tilde{v}_{r}^{1}+\varepsilon \tilde{v}_{r}^{2}, \tilde{v}_{z}=\tilde{v}_{z}^{0}+\varepsilon \tilde{v}_{z}^{1}, \tilde{p}:=\tilde{p}^{0}+\varepsilon \tilde{p}^{1} .
$$

Using our values we see that $R e=35$ and so the viscous coefficient is of order $1 / R e=0.03=\epsilon / 2$. The Strouhal number is $S h=61$.

Using (2) the asymptotic form of the contact force becomes

$$
\left(\left(p^{\varepsilon}-p_{r e f}\right) I-2 \mu D\left(v^{\varepsilon}\right)\right) \mathbf{n e}_{r}=\rho V^{2}\left(\tilde{p}-\tilde{p}_{r e f}+\mathcal{O}\left(\varepsilon^{2}\right)\right)\left(1+\frac{\Xi}{R} \tilde{\eta}\right) .
$$

In non-dimensional variables the deformed interface is defined by the equation $\tilde{r}=$ $1+\frac{\Xi}{R} \tilde{\eta}(\tilde{z}, \tilde{t})$. The leading-order equation for the coupling across the deformed lateral boundary describing continuity of forces and the continuity of velocity become

$$
\begin{gathered}
\frac{\rho V^{2}}{\mathcal{P}}\left(\tilde{p}-\tilde{p}_{r e f}+\mathcal{O}\left(\varepsilon^{2}\right)\right)\left(1+\frac{\Xi}{R} \tilde{\eta}\right)=\tilde{\eta}+\mathcal{O}\left(\varepsilon^{2}\right) \\
\tilde{v}_{r}\left(\tilde{z}, 1+\frac{\Xi}{R} \tilde{\eta}(z, t), \tilde{t}\right)=\frac{\partial \tilde{\eta}}{\partial \tilde{t}}, \quad \tilde{v}_{z}=0 .
\end{gathered}
$$

\subsection{The reduced two-dimensional coupled problem}

We summarize here the two-dimensional reduced coupled problem in non-dimensional variables. Define the scaled domain

$$
\tilde{\Omega}(\tilde{t})=\left\{(\tilde{z}, \tilde{r}) \in \mathbb{R}^{2} \mid \tilde{r}<1+\frac{\Xi}{R} \tilde{\eta}(\tilde{z}, \tilde{t}), 0<\tilde{z}<1\right\},
$$

and the lateral boundary $\tilde{\Sigma}(\tilde{t})=\left\{\tilde{r}=1+\frac{\Xi}{R} \tilde{\eta}(\tilde{z}, \tilde{t})\right\} \times(0,1)$. The problem consist of finding a $\left(\tilde{v}_{z}, \tilde{v}_{r}, \tilde{\eta}\right)$ such that in $\tilde{\Omega}(\tilde{t}) \times \mathbb{R}^{+}$the following is satisfied

$$
\begin{gathered}
S h \frac{\partial \tilde{v}_{z}}{\partial \tilde{t}}+\tilde{v}_{z} \frac{\partial \tilde{v}_{z}}{\partial \tilde{z}}+\tilde{v}_{r} \frac{\partial \tilde{v}_{z}}{\partial \tilde{r}}+\frac{\partial \tilde{p}}{\partial \tilde{z}}=\frac{1}{R e}\left\{\frac{1}{\tilde{r}} \frac{\partial}{\partial \tilde{r}}\left(\tilde{r} \frac{\partial \tilde{v}_{z}}{\partial \tilde{r}}\right)\right\}, \\
\frac{\partial}{\partial \tilde{r}}\left(\tilde{r} \tilde{v}_{r}\right)+\frac{\partial}{\partial \tilde{z}}\left(\tilde{r} \tilde{v}_{z}\right)=0, \\
\tilde{p}(\tilde{z}, \tilde{t})-\tilde{p}_{r e f}=\frac{\mathcal{P}}{\rho V^{2}} \frac{1}{\left(1+\frac{\Xi}{R} \tilde{\eta}\right)} \tilde{\eta}, \\
\tilde{v}_{r}\left(\tilde{z}, 1+\frac{\Xi}{R} \tilde{\eta}(z, t), \tilde{t}\right)=\frac{\partial \tilde{\eta}}{\partial \tilde{t}}, \quad \tilde{v}_{z}\left(\tilde{z}, 1+\frac{\Xi}{R} \tilde{\eta}(z, t), \tilde{t}\right)=0,
\end{gathered}
$$

with the initial and boundary conditions given by

$$
\begin{aligned}
& \tilde{v}_{r}=0 \text { and } \tilde{p}=\left(P_{1}(\tilde{t})+p_{r e f}\right) /\left(\rho V^{2}\right) \quad \text { on } \quad(\partial \tilde{\Omega}(\tilde{t}) \cap\{\tilde{z}=0\}) \times \mathbb{R}_{+} \text {, } \\
& \tilde{v}_{r}=0 \text { and } \tilde{p}=\left(P_{2}(\tilde{t})+p_{\text {ref }}\right) /\left(\rho V^{2}\right) \quad \text { on } \quad(\partial \tilde{\Omega}(\tilde{t}) \cap\{\tilde{z}=1\}) \times \mathbb{R}_{+} \text {, } \\
& \left.\tilde{\eta}\right|_{\tilde{t}=0}=\left.\frac{\partial \tilde{\eta}}{\partial \tilde{t}}\right|_{\tilde{t}=0}=0 ;\left.\quad \tilde{\eta}\right|_{\tilde{z}=0}=0, \quad \text { and } \quad \tilde{\eta} \mid \tilde{z}=1=0, \forall \tilde{t} \in \mathbb{R}_{+} \text {. }
\end{aligned}
$$


This is a closed, free-boundary problem for a two-dimensional degenerate hyperbolic system with a parabolic regularization. As in the simpler case of the rigid walls (see [Bre:99])we see that $\tilde{v}_{r}$ depends non-locally on $\tilde{v}_{z}$ and solving system (22)(28) is difficult both theoretically and numerically. Since our system generalizes the shallow water equations, it is customary to use a similar approach.

\subsection{The Reduced Equations with the Closure Hypothesis}

To simplify the problem even further and obtain the effective equations in one space dimension we use a typical approach of averaging the two-dimensional equations across the vessel cross-section. Introduce

$$
\begin{gathered}
\tilde{U}=\frac{2}{\left(1+\frac{\Xi}{R} \tilde{\eta}\right)^{2}} \int_{0}^{1+\frac{\Xi}{R} \tilde{\eta}} \tilde{v}_{z} \tilde{r} d \tilde{r}, \quad \tilde{\alpha}=\frac{2}{\left(1+\frac{\Xi}{R} \tilde{\eta}\right)^{2} \tilde{U}^{2}} \int_{0}^{1+\frac{\Xi}{R} \tilde{\eta}} \tilde{v}_{z}^{2} \tilde{r} d \tilde{r} \\
\tilde{A}=\left(1+\frac{\Xi}{R} \tilde{\eta}\right)^{2}, \quad \tilde{m}=\tilde{A} \tilde{U} .
\end{gathered}
$$

We integrate the incompressibility condition and the axial momentum equations with respect to $\tilde{r}$ from 0 to $1+\frac{\Xi}{R} \tilde{\eta}$ and obtain, after taking into account the no-slip condition at the lateral boundary,

$$
\frac{\partial \tilde{A}}{\partial \tilde{t}}+\frac{\Xi}{R} \frac{\partial \tilde{m}}{\partial \tilde{z}}=0, \quad S h \frac{\partial \tilde{m}}{\partial \tilde{t}}+\frac{\partial}{\partial \tilde{z}}\left(\tilde{\alpha} \frac{\tilde{m}^{2}}{\tilde{A}}\right)+\tilde{A} \frac{\partial \tilde{p}}{\partial \tilde{z}}=\frac{2}{R e} \sqrt{\tilde{A}}\left[\frac{\partial \tilde{v}_{z}}{\partial \tilde{r}}\right]_{\tilde{\Sigma}} .
$$

In the above system the Coriolis factor $\tilde{\alpha}$ depends on $v_{z}$ and the interface shear stress $\left[\frac{\partial \tilde{v}_{z}}{\partial \tilde{r}}\right]_{\tilde{\Sigma}}$ is unknown. A typical way of handling this problem in the theory of shallow water equation is to choose a closure, giving the dependence of $\tilde{\alpha}$ and $\left[\frac{\partial \tilde{v}_{z}}{\partial \tilde{r}}\right]_{\tilde{\Sigma}}$ on $\tilde{A}$ and $\tilde{m}$. The usual choice in the literature is

$$
\tilde{v}_{z}=\frac{\gamma+2}{\gamma} \tilde{U}\left(1-\left(\frac{\tilde{r}}{1+\frac{\Xi}{R} \tilde{\eta}}\right)^{\gamma}\right)
$$

(see [QTV:00]), with $\gamma=9$. We refer to [RobSeq:05] for the review of the closure formulas for the axial velocity $\tilde{v}_{z}$. Now the term on the right hand-side of the momentum equation becomes $-\frac{2}{R e}(\gamma+2) \frac{\tilde{m}}{\tilde{A}}$. After inserting the expression (24) for the pressure and returning to dimensional variables, we obtain the following quasilinear hyperbolic system

$$
\begin{gathered}
\frac{\partial A}{\partial t}+\frac{\partial m}{\partial z}=0 \\
\frac{\partial m}{\partial t}+\frac{\partial}{\partial z}\left(\alpha \frac{m^{2}}{A}\right)+\frac{A}{\rho} \frac{\partial}{\partial z}\left(R \mathcal{C} \sqrt{\frac{A_{0}}{A}}\left(\sqrt{\frac{A}{A_{0}}}-1\right)\right)=-\frac{2 \mu}{\rho}(\gamma+2) \frac{m}{A}
\end{gathered}
$$


where $A_{0}=R^{2}$. It is known that shocks do not form in system (30)-(31) for the realistic physiological parameters corresponding to a healthy human (see [CaKim:03]). Nevertheless, the weak point of the model is the closure hypothesis (29). It could introduce an error of order $\mathcal{O}(1)$ in the approximation and the parameter $\gamma$ is chosen to fit the experimental data. Moreover, the important Womersley flow could not be handled through (29). Our conclusion is that it would be of importance to get a closed model giving an approximation of order $\mathcal{O}\left(\varepsilon^{2}\right)$.

\section{$4 \mathrm{An} \varepsilon^{2}$-approximation without the ad hoc closure assumption}

In order to find a closure for the reduced problem, we are going to use homogenization theory. Homogenization theory is used to find effective equations for nonhomogeneous flows. For porous media problems it can be applied when (a) the pore size (characteristic size of the fluid region free of another phase) is smaller than a characteristic length of the macroscopic problem (here, vessel diameter) and (b) the pore includes a large number of molecules to be considered as continuum.

At a first glance using it in our setting is pointless. One should rather do a simple averaging of the equations for the fluid phase over the cross-section of the vessel. This approach is classical and we presented it in Section 3. It leads to an $\mathcal{O}\left(\varepsilon^{2}\right)$ approximation, but the resulting system (22)-(28) is very difficult to solve and its complexity was the reason for imposing an ad hoc velocity profile for the effective axial velocity.

But we know how to obtain nonlinear filtration laws in rigid periodic porous media by homogenization. In rigid periodic porous media the expansions are of lower order of precision, but we got a closed system. In this case it was possible to link the homogenized equations with the nonlinear algebraic relations between the pressure gradient and the velocity (Forchheimer's filtration law), found in experiments. For more details we refer to [MarMik:00] and [Mik:00]. We note that, in a similar way in [RobSeq:05], the equation (31) is replaced by a variant of Forchheimer's law linking $\frac{\partial \tilde{p}}{\partial \tilde{z}}$ with $\tilde{m}, \tilde{m}^{2}$ and $\frac{\partial \tilde{m}}{\partial \tilde{t}}$, and optimal approximations are derived for the case of rigid walls.

How to link the artery flow with the filtration through porous media ? Due to the uniform bound on the maximal value of the radial displacement, our artery could be placed into a rectangle with the length of order 1 and of the small width. By repeating periodically the geometry in the radial direction, we get a network of parallel, long and narrow tubes. This is one of the simplest porous media which one can imagine.

It is not a rigid but a deformable porous medium, as in Biot's theories of deformable porous media. All results which we could obtain for deformable porous media are also valid in our situation.

Motivated by the results from [MarMik:00] and [Mik:00], where closed effective porous medium equations were obtained using homogenization techniques, we would like to set up a problem that would mimic a similar scenario. In this vein, we introduce $y=\frac{1}{\varepsilon} \tilde{z}$ and assume periodicity in $y$ of the domain and of the veloc- 
ity and the pressure. Furthermore, recalling that we have a "thin" long tube with $\tilde{r}=\frac{1}{R} r=\frac{1}{\varepsilon} \frac{r}{L}$, we can assume periodicity in the radial direction thereby forming a network of a large number of strictly separated, parallel tubes. This now resembles a porous medium problem but with no flow from one horizontal tube to another. See Figure 4. We homogenize with respect to all directions. Since there is nothing in the

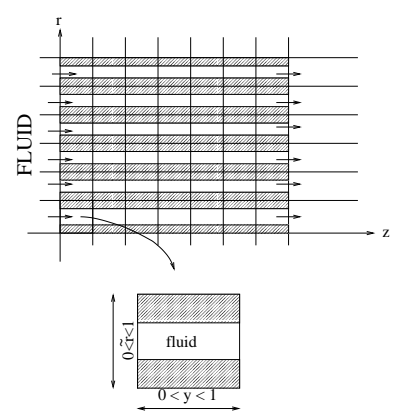

physics of the problem that depends periodically on $y$ we expect to get the effective equations and the solution independent of $y$.

More precisely, we start with the following relations between the "slow" variables $(r$ and $z$ (or $\tilde{z}))$ and "fast" variables $(\tilde{r}$ and $y$ )

$$
z=L \tilde{z}:=L \varepsilon y=R y, r=R \tilde{r} .
$$

The equations at zero order read

$$
\begin{gathered}
S h_{0} \frac{\partial \tilde{v}_{z}^{0}}{\partial \tilde{t}}+\left(\tilde{v}^{0} \nabla_{\tilde{r}, y}\right) \tilde{v}_{z}^{0}+\frac{\partial \tilde{\tilde{p}}^{0}}{\partial \tilde{z}}+\frac{\partial \tilde{\tilde{p}}^{1}}{\partial y}-\frac{1}{R e_{0}}\left\{\frac{1}{\tilde{\tilde{r}}} \frac{\partial}{\partial \tilde{r}}\left(\tilde{r} \frac{\partial \tilde{v}_{z}^{0}}{\partial \tilde{r}}\right)+\frac{\partial^{2} \tilde{v}_{z}^{0}}{\partial y^{2}}\right\}=0 \\
S h_{0} \frac{\partial \tilde{v}_{r}^{0}}{\partial \tilde{t}}+\left(\tilde{v}^{0} \nabla_{\tilde{r}, y}\right) \tilde{v}_{r}^{0}+\frac{\partial \tilde{\tilde{p}}^{0}}{\partial r}+\frac{\partial \tilde{\tilde{p}}^{1}}{\partial \tilde{r}}-\frac{1}{R e_{0}}\left\{\frac{1}{\tilde{r}} \frac{\partial}{\partial \tilde{r}}\left(\tilde{r} \frac{\partial \tilde{v}_{r}^{0}}{\partial \tilde{r}}\right)+\frac{\partial^{2} \tilde{v}_{r}^{0}}{\partial y^{2}}\right\}=0 \\
\nabla_{\tilde{r}, y} \tilde{\tilde{p}}^{0}=0 \\
\frac{\partial}{\partial \tilde{r}}\left(\tilde{r} \tilde{v}_{r}^{0}\right)+\frac{\partial}{\partial y}\left(\tilde{r} \tilde{v}_{z}^{0}\right)=0 \\
\tilde{v}_{r}^{0}, \tilde{v}_{z}^{0} \text { and } \tilde{\tilde{p}}^{1} \text { are 1-periodic in } y \text { and } \tilde{v}_{r}^{0}=\tilde{v}_{z}^{0}=0 \text { at } \tilde{r}=1+\frac{\Xi}{R} \tilde{\eta}
\end{gathered}
$$

where

$$
S h_{0}:=\frac{\varepsilon L \omega^{\varepsilon}}{V}, \quad R e_{0}:=\frac{\rho R V}{\mu} .
$$

Notice $S h_{0}=\varepsilon S h$ and $R e=\varepsilon R e_{0}$. For our values, $S h_{0}$ is of order $1\left(S h_{0} \in(3,4)\right)$ and $R e_{0}$ is around 600. We remark that equation (35) corresponds to the $\varepsilon^{-1}$ term. 
Here, a new scaling for the pressure was used to obtain equations (33)-(35). This "z-blown up" pressure scaling reads

$$
p=\frac{\rho L V^{2}}{R} \tilde{\tilde{p}}=\rho V^{2} \frac{1}{\varepsilon} \tilde{\tilde{p}}=\rho V^{2} \tilde{p}, \quad \text { so } \quad \tilde{\tilde{p}}=\varepsilon \tilde{p} .
$$

The leading order Navier equations for the membrane force are unchanged.

We now focus on the case corresponding to the magnitude of the parameters $S h_{0}$ and $R e_{0}$ which is of interest to us.

\subsection{Case with $S h>0$ and moderate $R e$}

In this case, for a given pressure gradient $\frac{\partial \tilde{\tilde{\tilde{x}}}^{0}}{\partial \tilde{\tilde{z}}}$, the non-stationary, axially symmetric system (33)-(37) admits a unique unidirectional, but strongly non-stationary solution. The unidirectional solution refers to the $y$ direction. We will write the solution of system (33)-(37) as a sum of this y-unidirectional solution and a small perturbation of it. This perturbation satisfies a linearized system, see (33)-(37), where the linearization is calculated around the unidirectional solution. This system is closed.

The zero-th order approximation: the y-unidirectional flow

For every given smooth $\tilde{\tilde{p}}^{0}$, system (33)-(37) has a unique strong solution

$$
\tilde{v}_{z}^{0}=w(\tilde{r}, \tilde{z}, t), \tilde{v}_{r}^{0}=0
$$

where $w$ satisfies

$$
\begin{gathered}
S h_{0} \frac{\partial w}{\partial \tilde{t}}-\frac{1}{R e_{0}} \frac{1}{\tilde{r}} \frac{\partial}{\partial \tilde{r}}\left(\tilde{r} \frac{\partial w}{\partial \tilde{r}}\right)=-\frac{\partial \tilde{\tilde{p}}}{\partial \tilde{z}}(\tilde{z}, \tilde{t})=-\frac{R \mathcal{C}}{\mathcal{P}} \frac{\partial \tilde{\eta}^{0}}{\partial \tilde{z}} \\
w(0, \tilde{z}, \tilde{t}) \text { bounded, } w\left(1+\Xi \tilde{\eta}^{0}(\tilde{z}, \tilde{t}) / R, \tilde{z}, \tilde{t}\right)=0 \quad \text { and } \quad w(\tilde{r}, \tilde{z}, 0)=0 .
\end{gathered}
$$

Furthermore, solution $\tilde{\tilde{p}}^{1}$ is a linear function of $y$, independent of $\tilde{r}$. Due to 1periodicity with respect to $y$ we get $\tilde{\tilde{p}}^{1}=\tilde{\tilde{p}}^{1}(\tilde{z}, \tilde{t})$. Then the next order in Laplace's law implies $\tilde{\tilde{p}}^{1}=0$. This is a free-boundary problem because the condition at the lateral boundary depends on the solution. For a known pressure or the radial displacement (or the cross-sectional area) this is well-posed. We can eliminate $\tilde{\tilde{p}}^{0}$, and use the definitions of $\tilde{A}$ and $\tilde{m}$ to write this in terms of $w$ and $\tilde{A}$ as

$$
\begin{gathered}
\frac{\partial \tilde{A}}{\partial \tilde{t}}+\frac{\Xi}{R} \frac{\partial}{\partial \tilde{z}} \int_{0}^{\sqrt{\tilde{A}}} 2 \tilde{r} w d \tilde{r}=0, \\
S h_{0} \frac{\partial w}{\partial \tilde{t}}+\left(\frac{R}{\Xi}\right)^{2} \frac{R}{L} \frac{\partial \sqrt{\tilde{A}}}{\partial \tilde{z}}=\frac{1}{R e_{0}} \frac{1}{\tilde{r}} \frac{\partial}{\partial \tilde{r}}\left(\tilde{r} \frac{\partial w}{\partial \tilde{r}}\right), \\
w(0, \tilde{z}, \tilde{t}) \text { bounded, } w(\sqrt{\tilde{A}}, \tilde{z}, \tilde{t})=0, \tilde{A}(\tilde{z}, 0)=0, w(\tilde{r}, \tilde{z}, 0)=0 \\
\tilde{A}(0, \tilde{t})=\tilde{A}(L, \tilde{t})=0 w(\tilde{r}, 0, \tilde{t})=w_{0}(t), w(\tilde{r}, L, \tilde{t})=w_{L}(t) .
\end{gathered}
$$

This is a two-dimensional, free-boundary problem of mixed, hyperbolic-parabolic type. It has a simpler form than system (19)-(20). 
The first-order correction: perturbation of the y-unidirectional flow

We will be using the zero-th order approximation to the solution consisting of the velocity $(w, 0)$ and displacement $\tilde{\eta}^{0}$ (or, equivalently, the pressure $\tilde{\tilde{p}}^{0}$ ) to find an $\varepsilon$ correction by solving (19)-(20), linearized around the zero-th order approximation:

$$
\begin{gathered}
S h_{0} \frac{\partial \tilde{v}_{z}^{1}}{\partial \tilde{t}}+\tilde{v}_{z}^{0}\left\{\frac{\partial \tilde{v}_{z}^{1}}{\partial y}+\frac{\partial \tilde{v}_{z}^{0}}{\partial \tilde{z}}\right\}+\tilde{v}_{r}^{1} \frac{\partial \tilde{v}_{z}^{0}}{\partial \tilde{r}}+\frac{\partial \tilde{\tilde{p}}^{2}}{\partial y}=\frac{1}{R e_{0}}\left\{\frac{1}{\tilde{r}} \frac{\partial}{\partial \tilde{r}}\left(\tilde{r} \frac{\partial \tilde{v}_{z}^{1}}{\partial \tilde{r}}\right)+\frac{\partial^{2} \tilde{v}_{z}^{1}}{\partial y^{2}}\right\} \\
S h_{0} \frac{\partial \tilde{v}_{r}^{1}}{\partial \tilde{t}}+\tilde{v}_{z}^{0} \frac{\partial \tilde{v}_{r}^{1}}{\partial y}+\frac{\partial \tilde{\tilde{p}}^{2}}{\partial \tilde{r}}=\frac{1}{R e_{0}}\left\{\frac{1}{\tilde{r}} \frac{\partial}{\partial \tilde{r}}\left(\tilde{r} \frac{\partial \tilde{v}_{r}^{1}}{\partial \tilde{r}}\right)+\frac{\partial^{2} \tilde{v}_{r}^{1}}{\partial y^{2}}\right\} \\
\frac{\partial}{\partial \tilde{r}}\left(\tilde{r} \tilde{v}_{r}^{1}\right)+\frac{\partial}{\partial y}\left(\tilde{r} \tilde{v}_{z}^{1}\right)+\frac{\partial \tilde{v}_{z}^{0}}{\partial \tilde{z}}=0 \\
\tilde{v}_{r}^{1}, \tilde{v}_{z}^{1}, \tilde{\tilde{p}}^{2} \text { are 1-periodic in } y ; \tilde{v}_{r}^{1}=\frac{\partial \tilde{\eta}^{0}}{\partial \tilde{t}}, \tilde{v}_{z}^{0}=0 \text { at } \tilde{r}=1+\frac{\Xi}{R} \tilde{\eta}^{0}
\end{gathered}
$$

This is an Oseen's system and it has a $\tilde{v}^{1}$ is uniquely determined. We search $\tilde{v}_{z}^{1}=$ $\tilde{v}_{z}^{1}(\tilde{r}, \tilde{z}, \tilde{t})$. Then

$$
\tilde{r} \tilde{v}_{r}^{1}(\tilde{r}, \tilde{z}, \tilde{t})=\left(1+\Xi \tilde{\eta}^{0} / R\right) \frac{\partial \tilde{\eta}^{0}}{\partial \tilde{t}}+\int_{\tilde{r}}^{1+\Xi \tilde{\eta}^{0} / R} \frac{\partial \tilde{v}_{z}^{0}}{\partial \tilde{z}}(\xi, \tilde{z}, \tilde{t}) \xi d \xi .
$$

Furthermore

$$
\begin{gathered}
S h_{0} \frac{\partial \tilde{v}_{z}^{1}}{\partial \tilde{t}}-\frac{1}{R e_{0}} \frac{1}{\tilde{r}} \frac{\partial}{\partial \tilde{r}}\left(\tilde{r} \frac{\partial \tilde{v}_{z}^{1}}{\partial \tilde{r}}\right)+\frac{\partial \varphi}{\partial y}(y, \tilde{z}, \tilde{t})=-\tilde{v}_{r}^{1} \frac{\partial \tilde{v}_{z}^{0}}{\partial \tilde{r}}-\frac{\partial}{\partial \tilde{z}}\left(\frac{\left(\tilde{v}_{z}^{0}\right)^{2}}{2}+\tilde{\tilde{p}}^{1}\right) \\
\tilde{v}_{z}^{1}(0, \tilde{z}, \tilde{t}) \text { is bounded, } \quad \tilde{v}_{z}^{1}\left(1+\Xi \tilde{\eta}^{0}(\tilde{z}, \tilde{t}) / R, \tilde{z}, \tilde{t}\right)=0 \\
\tilde{v}_{z}^{1}(\tilde{r}, \tilde{z}, 0)=0 \quad \text { and } \quad \tilde{\tilde{p}}^{2}=\tilde{\tilde{p}}(\tilde{r}, \tilde{z}, \tilde{t})
\end{gathered}
$$

This solution also satisfies problem (22)-(48) to $\varepsilon^{2}$-order. More precisely, since $\tilde{\tilde{p}}^{0}=$ $\varepsilon \tilde{p}$ and due to the boundary conditions for the pressure, we have that $\tilde{\tilde{p}}^{0}$ is of order $\varepsilon$. Consequently, both $\tilde{v}_{z}^{0}$ and $\tilde{v}_{r}^{1}$ are of order $\varepsilon$. We have

Proposition 2. The velocity field $\left(\tilde{v}_{z}^{0}+\varepsilon \tilde{v}_{z}^{1}\right)$ and the pressure field $\frac{1}{\varepsilon} \tilde{\tilde{p}}^{0}$ satisfy equations (22)-(24) to $\mathcal{O}\left(\varepsilon^{2}\right)$.

\section{Conclusion: The problem with nonlinear coupling in dimensional form}

Using the homogenization approach we obtained a closed "one-and-a-half-dimensional" effective model, which approximates the system (1)-(7), in the limit $\varepsilon \rightarrow 0$. We did 
not need any ad hoc closure assumptions. Our limit system could be classified as a special case of the diphasic Biot's system (see [Biot:56]. Its numerical solution is much simpler than that of the shallow water systems. For detailed presentation of the numerical simulations we refer to [CLMT:05] and to [CMT:05]. We repeat once more our construction, but this time in dimensional form.

The zero-th order approximation

Look for $v_{z}^{0}=v_{z}^{0}(r, z, t)$ and $\eta^{0}=\eta^{0}(z, t)$ and then recover $p^{0}=p^{0}(z, t)$ by solving the following free-boundary problem defined on the domain $0<z<L$, $0<r<R+\eta^{0}(z, t)$

$$
\begin{gathered}
\frac{\partial\left(R+\eta^{0}\right)^{2}}{\partial t}+\frac{\partial}{\partial z} \int_{0}^{R+\eta^{0}} 2 r v_{z}^{0} d r=0 \\
\rho \frac{\partial v_{z}^{0}}{\partial t}+\frac{\partial p}{\partial z}=\mu \frac{1}{r} \frac{\partial}{\partial r}\left(r \frac{\partial v_{z}^{0}}{\partial r}\right), \\
v_{z}^{0}(0, z, t) \text { bounded, } v_{z}^{0}\left(R+\eta^{0}(z, t), z, t\right)=0, \quad v_{z}^{0}(r, z, 0)=0
\end{gathered}
$$

with the following inlet and outlet boundary conditions $\forall t \in \mathbb{R}_{+}$

$$
\begin{gathered}
\eta^{0}=0 \text { for } z=0, \eta^{0}=0 \text { for } z=L, \\
p=P_{1}(t)+p_{\text {ref }} \text { for } z=0,0 \leq r \leq R, \\
p=P_{2}(t)+p_{\text {ref }} \text { for } z=L, 0 \leq r \leq R,
\end{gathered}
$$

with the pressure

$$
p(z, t)=p_{r e f}+R \mathcal{C} \frac{\eta^{0}}{R+\eta^{0}} .
$$

The $\varepsilon$-correction for the velocity

Solve for $v_{z}^{1}=v_{z}^{1}(r, z, t)$ and $v_{r}^{1}=v_{r}^{1}(r, z, t)$ by first recovering $v_{r}^{1}$ via

$$
r v_{r}^{1}(r, z, t)=\left(R+\eta^{0}\right) \frac{\partial \eta^{0}}{\partial t}+\int_{r}^{R+\eta^{0}} \frac{\partial v_{z}^{0}}{\partial z}(\xi, z, t) \xi d \xi
$$

and then solve the following linear fixed boundary problem for $v_{z}^{1}$, defined on the domain $0<z<L, 0<r<R+\eta^{0}(z, t)$

$$
\begin{gathered}
\frac{\partial v_{z}^{1}}{\partial t}-\nu \frac{1}{r} \frac{\partial}{\partial r}\left(r \frac{\partial v_{z}^{1}}{\partial r}\right)=-S_{v_{z}^{1}}(r, z, t) \\
v_{z}^{1}(0, z, t) \text { bounded, } v_{z}^{1}\left(R+\eta^{0}(z, t), z, t\right)=0 \\
v_{z}^{1}(r, 0, t)=v_{z}^{1}(r, L, t)=0 \quad v_{z}^{1}(r, z, 0)=0
\end{gathered}
$$

where $S_{v_{z}^{1}}(r, z, t)$ contains the already calculated functions and is defined by 


$$
S_{v_{z}^{1}}(r, z, t)=v_{r}^{1} \frac{\partial v_{z}^{0}}{\partial r}+v_{z}^{0} \frac{\partial v_{z}^{0}}{\partial z} .
$$

The result of Proposition 2 implies that the velocity field $\left(v_{z}^{0}+\varepsilon v_{z}^{1}\right)$ and the pressure field $p$ represent a second order approximation for the solutions of the equations (1)-(7).

\section{References}

[BdV:04] H. Beirao da Veiga : On the existence of a strong solution to a coupled fluidstructure evolution problem, Journal of Mathematical Fluid Mechanics, Vol. 6 (2004), p. 21-52.

[Biot:56] M.A. Biot. Theory of propagation of elastic waves in a fluid-saturated porous solid. I. Lower frequency range, and II. Higher frequency range, J. Acoust. Soc. Am. 28(2) (1956), 168-178 and 179-191.

[Bre:99] Y. Brenier: Homogeneous hydrostatic flows with convex velocity profiles. Nonlinearity, Vol. 12 (1999), p. 495-512.

[CaKim:03] S. Čanić and E-H. Kim. Mathematical analysis of the quasilinear effects in a hyperbolic model of blood flow through compliant axi-symmetric vessels. Mathematical Methods in the Applied Sciences, 26(14) (2003), 1161-1186.

[CLMT:05] S. Čanić, D. Lamponi, A. Mikelić , J. Tambača : Self-Consistent Effective Equations Modeling Blood Flow in Medium-to-Large Compliant Arteries, SIAM Journal on Multiscale Analysis and Simulation, Vol. 3 (2005), p. 559-596.

[CMT:05] S. Čanić, A. Mikelić , J. Tambača : A Two-Dimensional Effective Model Describing Fluid-Structure Interaction in Blood Flow: Analysis, Numerical Simulation and Experimental Validation, to appear in Comptes Rendus Mécanique , 2005.

[DEGLT:01] B. Desjardins, M.J. Esteban, C. Grandmont, P. Le Tallec, Weak solutions for a fluid-structure interaction model, Rev. Mat. Complut. 14(2001), p. 523 - 538

[Fung:93] Y.C. Fung. Biomechanics: Circulation. Springer New York (1993). Second Edition.

[MarMik:00] E. Marušić-Paloka and A. Mikelić. The derivation of a nonlinear filtration law including the inertia effects via homogenization. Nonlinear Analysis 42 (2000), 97-137.

[Mik:00] A. Mikelić. Homogenization theory and applications to filtration through porous media, chapter in "Filtration in Porous Media and Industrial Applications," by M. Espedal, A. Fasano and A. Mikelić, Lecture Notes in Mathematics Vol. 1734, Springer, 2000, 127-214.

[Nobile:01] F. Nobile: Numerical Approximation of Fluid-Structure Interaction Problems with Application to Haemodynamics. Ph.D. Thesis, EPFL, Lausanne (2001).

[RobSeq:05] A.M. Robertson, A. Sequeira: A director theory approach for modeling blood flow in the arterial system: an alternative to classical 1D models, $\mathrm{M}^{3}$ AS : Math. Models Methods Appl. Sci., Vol. 15 (no. 6)) (2005), p. 871-906.

[QTV:00] A. Quarteroni, M. Tuveri and A. Veneziani. Computational vascular fluid dynamics: problems, models and methods. Survey article, Comput. Visual. Sci. 2 (2000), 163-197. 